\title{
Time-dependent displacement of commensal skin microbes by pathogens at the site of colorectal surgery
}

\section{Jennifer Holder-Murray}

University of Pittsburgh Medical Center

\section{Andrew Yeh}

University of Pittsburgh Medical Center

\section{Matthew Rogers}

University of Pittsburgh School of Medicine

\section{Brian Firek}

University of Pittsburgh School of Medicine https://orcid.org/0000-0001-9908-8214

\section{Brandon Mahler}

University of Pittsburgh Medical Center

\section{David Medich}

University of Pittsburgh Medical Center

James Celebrezze

University of Pittsburgh Medical Center

Michael Morowitz ( $\square$ michael.morowitz@chp.edu )

https://orcid.org/0000-0001-8172-637X

\section{Research}

Keywords: microbiome, colorectal surgery, wound infection, cutaneous

Posted Date: June 10th, 2020

DOI: https://doi.org/10.21203/rs.3.rs-34436/v1

License: (c) (i) This work is licensed under a Creative Commons Attribution 4.0 International License. Read Full License 


\section{Abstract}

Objective: To characterize perioperative changes of the skin microbiome in patients undergoing elective colorectal surgery and to determine this relationship with surgical site infections (SSIs).

Summary of Background Data: Despite the prevalence and significance of SSIs, their pathogenesis remains poorly understood. Although the complexity of the human skin microbiome has been the subject of recent studies, it is not known whether alterations among commensal microbes contribute to the incidence of SSIs.

Methods: Skin swabs of the abdominal wall and chest wall from 60 study subjects were collected before and after colorectal surgery, in addition to intraoperative samples including subcutaneous fat and colonic contents. Bacterial 16S rRNA gene sequences were sequenced and analyzed.

Results: Alpha diversity on the skin decreased in the perioperative period but later recovered at the postoperative clinic visit. Alpha diversity of the subcutaneous fat increased significantly between the beginning and end of these operations, with an increase in abundance of gut microbes also seen within luminal contents after colon resection. In the early postoperative period, the abundance of Enterobacteriaceae increased at the site of surgical incision, with a concomitant decrease in commensals including Corynebacterium and Propionibacterium. Only one patient developed a wound infection. Incisional skin swabs from this patient demonstrated a sharp postoperative increase in the abundance of Enterococcus, which was later cultured from wound drainage at the time of SSI diagnosis.

Conclusions: In most patients, we observed a transient postoperative loss of skin commensals at the surgical site, which were replaced by potential pathogens and anaerobes from the gut.

We postulate that real time monitoring of the skin microbiome, in parallel with improved knowledge with the gut microbiome and gastrointestinal surgery, could provide actionable findings about the pathogenesis of SSIs.

\section{Introduction}

Surgical site infections (SSIs) remain an expensive cause of morbidity after colorectal surgery ${ }^{1}$. It is commonly said that SSIs are preventable, but this idea remains largely unproven ${ }^{2}$. This notion can be traced back to speculations from Lister and Halsted that infections result from wound contamination ${ }^{3}$. Today, the problem of SSIs persists despite the ubiquity of novel products developed to maintain intraoperative sterility and barriers. Just in the last decade, studies have assessed the role of preoperative skin preparation ${ }^{4}$, antibiotic coated sutures ${ }^{5}$, adhesive drapes ${ }^{6}$, wound protectors ${ }^{7}$, and absorbable antibiotic coated sponges ${ }^{8}$ left within wounds at closure. The failure to eradicate SSIs suggests that our understanding of SSI pathogenesis is incomplete. 
It is generally recognized that infections represent a context-dependent host-microbe interaction ${ }^{9}$, but this relationship is not well understood with regard to SSI pathogenesis. The focus on contamination may underestimate the importance of host factors and non-microbial environmental factors. The outsized emphasis on reducing intraoperative contamination may be because it is easier to operationalize than interventions regarding the myriad genetic and environmental determinants of surgical outcomes. Still, it is known that some patient characteristics increase the risk of SSIs, including inflammatory bowel disease ${ }^{10}$, obesity ${ }^{11}$, smoking ${ }^{12}$, and procedures such as ERCP $^{13}$. It is not fully known why such host factors increase the risk of SSI.

The skin microbiome is a host factor that may play an underappreciated role in the pathogenesis and prevention of wound infections. Knowledge of the skin microbiota lags behind the gut ${ }^{14}$, but the existing literature makes it clear that the skin harbors clinically relevant microbial communities ${ }^{15,16}$ that interact with the host immune system ${ }^{16}$. Recent experimental studies have demonstrated contributions of skin microbes to neoplasia ${ }^{17}$, inflammation ${ }^{18}$, and the pathogenesis of infection. Other studies suggest that wound healing and inflammation are also impacted by both commensal and pathogenic skin bacteria ${ }^{22-}$ 27.

To date, investigations of the microbiome of human wounds have focused upon chronic wounds, e.g. diabetic ulcers. Few studies have chronicled the bacteriology of surgical incisions ${ }^{28-31}$. In this study, we utilized a high-throughput approach to characterize the skin microbiota of adults undergoing colorectal surgery. To our knowledge, there are no other studies of this subject in colorectal surgery, where the incidence of SSI remains as high as $20 \%$ in some studies ${ }^{32}$. We collected a large number of skin swabs and fecal samples during the perioperative period to clarify perioperative changes in the skin microbiota. Additionally, we sought to determine if postoperative skin dysbiosis contributed to SSI.

\section{Methods}

\section{Patient Selection}

Adults undergoing outpatient evaluation prior to colorectal surgery at University of Pittsburgh Medical Center were recruited to participate in this study (University of Pittsburgh PR015080161) from January 2016 through March 2018. Patients were eligible for participation if they were scheduled to undergo resection of the small or large intestine.

\section{Patient Factors}

All patients underwent surgery via an enhanced recovery protocol ${ }^{33}$ after administering a chlorhexidine bath on the night before surgery. All subjects undergoing total colon or left colon surgery or proctectomy underwent mechanical bowel preparation prior to surgery; subjects undergoing right or transverse colon surgery underwent mechanical bowel preparation based on surgeon preference. No oral antibiotic regimen was used based on institutional protocol except for six patients early in the study who received 
neomycin and metronidazole. Chlorhexidine solution was used as intraoperative skin preparation unless an allergy had been noted. Preoperative intravenous antibiotics (ceftriaxone and metronidazole) were administered; if necessary, appropriate antibiotic prophylaxis was chosen based on patient allergy profile. During surgery, laparotomy or extraction site wound protectors were utilized unless the incision was too large to accommodate the device. All operative personnel changed gloves and used clean surgical instruments for abdominal wall closure. Following fascial closure, subcutaneous tissues were irrigated with sterile saline. Sterile wound dressings of gauze with tape were left in place until postoperative day 2; following dressing removal, incisions were redressed only in the presence of wound seepage or if the incision was in a pannus fold. Signs of wound infection (erythema, purulent drainage) were assessed daily by the clinical team.

\section{Sample Collection and Preparation of Bacterial DNA for $16 S$ rRNA amplicon sequencing}

For a detailed description of sample collection and microbiome analyses, see eMethods. Briefly, skin swabs of the largest abdominal incision and of the anterior chest wall were collected at preoperative clinic visits, during the perioperative hospitalization, and at postoperative clinic visits. Rectal swabs, fecal samples, and/or ileostomy effluent were collected during the perioperative hospitalization. Additional sample types included intestinal contents harvested from the surgical specimen, swabs of the subcutaneous tissue during opening and closing of the abdominal incision, and wound drainage if present.

\section{Results}

\section{Patient Characteristics}

The study included 60 adults ( $58 \%$ female, mean age 58 years) that underwent elective intestinal resection for colorectal adenocarcinoma (22 patients), diverticular disease (12 patients), and inflammatory bowel disease (8 patients). The most common surgeries performed (Table 1) in this cohort included laparoscopic right hemicolectomy / ileocecectomy (20 patients), laparoscopic sigmoidectomy (14 patients), and laparoscopic total abdominal colectomy (10 patients). 
Table 1

Clinical characteristics of study cohort.

\begin{tabular}{|ll|}
\hline Patient Demographics & 60 \\
\hline Total patients & $58(59,22$ to 86$)$ \\
\hline Age & 35 \\
\hline Number of females & 4.4 days (3.6 days, 1.7 days to 20.1 days) \\
\hline Length of stay & 29 to 22 to 9 \\
\hline Left:right:total & 52 to 8 \\
\hline Lap:open & 25 to 35 \\
\hline cancer:non-cancer & 17 (polyethylene glycol) \\
\hline mech bowel prep & 12 \\
\hline Indication for Surgery & 2 \\
\hline diverticular disease & 20 \\
\hline rectal adenocarcinoma & 1 \\
\hline colon adenocarcinoma & 7 \\
\hline appendiceal adenocarcinoma & 1 \\
\hline Crohn's disease & 1 \\
\hline benign polyp & 5 \\
\hline ulcerative colitis & 1 \\
\hline Lynch & 1 \\
\hline FAP & 1 \\
\hline lymphoma & \\
\hline neuroendocrine tumor & \\
\hline sarcoma & 1 \\
\hline
\end{tabular}

\section{Comparison of microbial communities across body sites}

We collected 990 total samples from 60 patients. Of these, 882 samples (eTable 1) passed quality filtering after sequencing and amplification. As expected, alpha diversity (observed OTUs metric) was higher in fecal samples, rectal swabs, and colonic luminal contents than in skin swabs (eFigure 1). Among skin swabs, alpha diversity was similar between chest swabs and abdominal swabs at all time points except during the hospital stay (Fig. 1). On POD 1 and 2 we observed significantly lower alpha diversity within abdominal swabs. Interestingly, alpha diversity did not differ significantly before and after 
skin preparation with chlorhexidine $(p=0.2616)$. However, 16 S rRNA analyses of bacterial DNA cannot discern between live and dead bacterial cells, so this does not necessarily indicate that skin decontamination was inadequate.

As also expected, beta diversity analysis (principle coordinate analysis of community composition) demonstrated that skin samples formed a discrete group from fecal samples and luminal contents, but considerable overlap existed between abdominal skin swabs and chest wall swabs for either preoperative, hospital stay or postoperative clinic visit (Fig. 2A). Differences between sites were significant by PERMANOVA analysis, as were pairwise PERMANOVA comparisons between sites, though with only modest $R$-squared values (Abdomen vs Fecal, $R^{2}=0.15437$; Chest vs Fecal, $R^{2}=0.21417$ ).

\section{Temporal changes in the perioperative microbiome}

Among chest swabs, alpha diversity decreased during the time between preoperative clinic visit and day of surgery, perhaps reflecting the self-administered chlorhexidine bath on the night before surgery. After surgery, alpha diversity in chest swabs quickly returned to preoperative levels despite perioperative antibiotics and subsequently did not change significantly (Fig. 1). By contrast, alpha diversity near the abdominal incision decreased on the day of surgery, remained significantly lower during hospitalization until day 3 , and slowly returned to normal by the postoperative visit (Fig. 1).

Beta diversity analyses indicated that preoperative clinic, perioperative, and postoperative clinic skin swabs overlap considerably in PCoA space when visualized as a scatter plot. This was true for swabs of both the abdomen (Fig. 2B) and the chest (Fig. 2C). Another way to visualize differences in beta diversity differences between time points is to consider the distribution of weighted UniFrac distances between abdominal skin sample groups. We found that, while distances between sample group comparisons were small, the distances between preoperative and postoperative clinic samples were even smaller than distances of either group to postoperative hospital stay samples, though these statistical differences did not reach significance (Fig. 3A).

\section{Intraindividual variation of skin microbiome}

To quantify the extent of variation within patients, we calculated distances for each subject between preoperative samples, postoperative clinic samples, and the earliest postoperative hospital stay sample (Fig. 3B). Interestingly, we found that preoperative and postoperative clinic visits samples from the same patient were more similar than comparisons between all preoperative clinic (all patients) and all postoperative clinic visits samples (all patients), illustrating the resilience and personalized nature of the skin microbiome even in the perioperative period.

We also tracked UniFrac distances over time for abdominal and chest skin samples by comparing samples from each patient against a common reference point (preoperative samples from the same patient) (Fig. 3C). All patients' samples diverged considerably from their preoperative clinic visit sample, and in abdomen skin samples this distance peaked by POD 2 indicating that this is where the abdominal 
microbiome became most perturbed. Chest skin samples did not show the same degree of variation over time and were on average more similar to the preoperative samples.

To assess wound contamination during the surgery, we investigated swabs of subcutaneous fat at the beginning and end of the operation. Indeed, alpha diversity of the subcutaneous fat increased between the beginning and end of these operations, indicating an increase in bacterial load (eFigure 2, $p=$ 0.03524). In fact, many subcutaneous fat samples from the beginning of surgery did not pass quality control filtering for sequencing data, presumably because so few bacterial cells were present. By contrast, swabs collected at closure tended to sequence well and possessed microbial communities relatively similar to intestinal samples. Beta diversity (community composition) of the subcutaneous swabs collected at the beginning and the end of the operation differed significantly (PERMANOVA $p$-value $<0.05$ ) (not shown).

We also compared weighted UniFrac distances of the closure swabs to colonic contents of the same patient and from other patients. The subcutaneous fat microbiome of the extraction incision immediately after opening was highly dissimilar from luminal contents sampled intraoperatively (eFigure 2), regardless of whether the lumen contents were from the same patient or from other subjects. The same was not true for the subcutaneous fat swabs collected at the time of wound closure of the incision. Rather, we found that the subcutaneous fat microbiome at closure was surprisingly similar to luminal contents sampled intraoperatively from the same patient but not contents from other patients (eFigure 2; $\mathrm{P}=1.776 \mathrm{e}-15)$. This result provides evidence that microbes from the lumen of the resected colon colonize the wound during the surgery, although these microbes are not necessarily those involved in SSI pathogenesis several days later.

\section{Analysis of Taxonomic Composition}

We found multiple taxa within abdominal skin swabs that differed across time points, some of which were present in high abundance in some subjects. In the first two days following surgery, the abundance of Enterobacteriaceae increased, with a concomitant decrease in Corynebacteriaceae (Fig. 4A and eTable 2). It is known that amplification and sequencing of the V4 region of bacterial $16 \mathrm{~S}$ rRNA genes is not accurate in measuring the abundance of the common skin genus Propionibacterium. For this reason, we utilized qPCR with Propionibacterium specific primers to estimate its abundance (Fig. 4B). Like Corynebacterium, the abundance of Propionibacterium also decreased considerably after surgery. We found few changes in comparisons between preoperative clinic visit and postoperative chest swabs.

In agreement with beta diversity analyses of subcutaneous swabs, taxonomic analysis demonstrated a remarkably increased abundance of colonic anaerobes Lachnospiraceae and Bacteroidaceae in the extraction incision at closure but not at opening (Fig. 4C and eTable 2). We evaluated whether patterns of microbial diversity were associated with specific clinical variables. However, we did not identify significant differences in the skin microbiota based upon gender, age, antibiotic exposure, mode of preoperative bowel regimen, presence of cancer, or the side (right or left) of the colorectal resection (data not shown). 


\section{Postoperative infection}

Postoperatively, only one patient developed a wound infection. No other patients required their wounds to be opened for any reason. The study subject with an SSI (Patient 8) grew Enterococcus faecalis on a swab of wound drainage sent to the microbiology laboratory on POD 11. Interestingly, Enterococcus was not present in skin swabs at the preoperative clinic visit, before or after skin preparation on the day of surgery (eFigure 3), subcutaneous fat swabs of the extraction incision at the beginning or end of surgery, or incision swabs on the first two days after surgery. However, by POD 4, Enterococcus was seen at high abundance in ileostomy effluent and also at low abundance in abdominal skin swabs. The abundance on the abdominal skin swabs then increased progressively and ultimately Enterococcus was identified in drainage from the wound both by culture and by $16 \mathrm{~S}$ rRNA gene sequencing.

The postoperative appearance of Enterococcus on the wound also corresponded with a decrease in the abundance of skin commensals Corynebacterium, Staphylococcus, and Propionibacterium. These common skin commensals were each seen preoperatively, then disappeared in the postoperative period before partially reappearing on abdominal skin swabs later in the hospital stay and at the postoperative clinic visit. Enterococcus was still detectable on the skin at the postoperative visit when the wound infection was resolved, but at that time it was no longer detectable in the ileostomy effluent.

\section{Discussion}

Most abundant skin organisms are likely unknown to most clinicians and operating room staff. Indeed, preoperative skin preparation and antibiotic administration should be expected to obliterate the skin microbiota but the clinical consequences of these interventions are understudied. We postulate that improved knowledge of the skin microbiome and the associated immune response, in parallel with improved knowledge of the gut microbiome and gastrointestinal surgery, could provide actionable findings about SSI pathogenesis.

The purpose of this study was to characterize changes in the cutaneous microbiome in patients undergoing major elective colorectal procedures for both benign and malignant disease. Swabs of the chest wall served as internal controls for postoperative swabs of the incision. To our knowledge, this is the largest study to date of the microbiota of surgical incisions, and the samples collected were paired with intestinal contents, stool samples, and intraoperative swabs of the subcutaneous fat in the wounds. Overall, as expected, we observed a skin microbiota marked by an abundance of Corynebacteriaceae, Propionibacterium, and Staphylococcus.

The key finding was a transient postoperative loss of the skin commensals Corynebacterium and Propionibacterium and a transient colonization by potential pathogens as well as anaerobes from the gut. This appears to be unique to the surgical site, as the observed chest microbiota was far more stable. During the period of microbial instability after surgery, skin swabs contained unusually high levels of Enterobacteriaceae and intestinal anaerobes such as Lachnospiraceae and Bacteroidaceae. Interestingly, 
the swabs of the subcutaneous fat from the opening incisions did not demonstrate complex microbial communities, whereas swabs at wound closure contained an intestine-like microbiota.

Because the SSI rate in this cohort was low, it was not possible to formally test the hypothesis that pathogens responsible for SSIs would be present on postoperative swabs prior to onset of infection. This lack of SSIs may reflect the large number of minimally invasive cases, which generally enjoy lower complication rates ${ }^{34}$. In the single patient with an Enterococcus wound infection, we observed a dramatic increase in abundance of Enterococcus in ileostomy effluent and concomitantly on swabs of the abdominal skin. This coincided almost perfectly with a loss of the commensals. The fact that the pathogen responsible for wound infection was not seen in the luminal contents or subcutaneous fat swabs supports the concept that wound infection may not necessarily reflect intraoperative contamination. It may be the case that Enterococcus bloomed in the setting of antibiotic exposure ${ }^{35}$, and subsequently spread to cause infection.

Although we did not observe a signal linking skin microbiome and SSI, the available literature would suggest that our findings are clinically relevant. The loss of skin commensals near the wound likely creates an opportunity for growth of pathogens and other organisms not typically found on the skin, as seen also in models of skin wounds ${ }^{27,31}$. The observed dysbiosis, like dysbiosis at other body locations, brings about two related problems. First, affected individuals acutely suffer from the loss of commensals that may be important for health and immune function. Second, with commensals absent, potential pathogens may grow in a compensatory fashion in a manner akin to loss of colonization resistance, which has been better studied in the intestine ${ }^{36,37}$. Clearly, since most subjects in this study did not suffer complications despite displaying postoperative skin dysbiosis, additional variables must be responsible for the pathogenesis of SSIs. Identifying these variables and integrating them with knowledge of postoperative dysbiosis could be useful in preventing SSI.

If larger studies in the future allow for identification of colonization patterns associated with onset of infection, then there may be actionable opportunities for improved perioperative care. First, it may be possible to identify surgical incisions at high risk of infection, and then monitor the microbiome of these incisions over time. Early identification of postoperative skin dysbiosis may then allow for preventive measures to restore a normal skin microbiota. For example, topical probiotic therapy has now been embraced as a method to optimize the skin microbiome ${ }^{38,39}$. Other available interventions to modulate the skin microbiota include topical application of honey-based ointments or the use of enteral probiotics. Proof-of-principle for perioperative manipulation of the microbiome was provided with the demonstration that administration of mupirocin for patients found to be colonized preoperatively with methicillinresistant $S$. aureus improves outcomes ${ }^{40}$.

Finally, this study raises questions about the unfocused approach to obliterate the perioperative skin microbiome in the hopes of preventing SSI. Minimizing intraoperative contamination will always be important, but could be integrated with microbiome science. Ultimately, when a wound becomes infected and a causative bacterial species is isolated from culture, one might attempt to determine the origin of 
the offending organism. If the operating room environment is identified as the source of infection, then the focus on sterility is appropriate. If the offending organism is found to be a member of the patient's native microbiome or the microbiome that emerges in the setting of perioperative antibiotics, then the focus on sterility may be a distraction. This is particularly true in the case of clean operations. For example, it is puzzling to see sternotomy or laminectomy incisions infected with intestinal organisms even without violation of the gastrointestinal tract ${ }^{41,42}$. Larger and more comprehensive perioperative studies of the microbiota could begin to answer these questions and, hopefully, improve patient outcomes.

\section{Declarations}

Ethics approval and consent to participate

Adults undergoing outpatient evaluation prior to colorectal surgery at University of Pittsburgh Medical Center were recruited to participate in this study from January 2016 through March 2018 under the University of Pittsburgh Institutional Review Board protocol number PRO15080161.

\section{Consent for publication}

Not applicable

\section{Availability of data and materials}

The DNA sequencing data used in the current study are available in the NCBI BioProject data repository, accession number PRJNA635382. The OTU table, OTU sequences, and code are available on GitHub at https://github.com/MattRogers2/CRS.

\section{Competing interests}

The authors declare that they have no competing interests.

\section{Funding}

This project was partially funded by a Limited Project Grant from the American Society of Colon and Rectal Surgeons Research Foundation (LPG-105). 
J.H.M. and M.J.M conceived the study. J.H.M., A.Y., B.M. and M.J.M and planned the human subjects research. J.H.M., B.M., A.Y., D.M., and J.C. obtained samples and metadata. B.F. prepared and submitted all samples for sequencing. A.Y., M.R., and M.J.M. analyzed all data. J.H.M., A.Y., M.R., and M.J.M. contributed to interpretation of the results and co-wrote the manuscript. All authors provided critical feedback and helped shape the research, analysis and manuscript.

\section{Acknowledgements}

We would like to thank Dr. Andrew Watson and Dr. Javier Salgado, acknowledging their contributions to patient enrollment and perioperative sample collection.

\section{References}

1. Lipsett PA. Surgical Site Infection Prevention-What We Know and What We Do Not Know. JAMA Surg. 2017;152(8):791-2. doi:10.1001/jamasurg.2017.0901.

2. Berríos-Torres SI, Umscheid CA, Bratzler DW, et al. Centers for Disease Control and Prevention Guideline for the Prevention of Surgical Site Infection, 2017. JAMA Surg. 2017;152(8):784-91. doi:10.1001/jamasurg.2017.0904.

3. Gaines S, Luo JN, Gilbert J, Zaborina O, Alverdy JC. Optimum Operating Room Environment for the Prevention of Surgical Site Infections. Surg Infect. 2017;18(4):503-7. doi:10.1089/sur.2017.020.

4. Darouiche RO, Wall MJ, Itani KMF, et al. Chlorhexidine-Alcohol versus Povidone-lodine for SurgicalSite Antisepsis. N Engl J Med. 2010;362(1):18-26. doi:10.1056/NEJMoa0810988.

5. Elsolh B, Zhang L, Patel SV. The Effect of Antibiotic-Coated Sutures on the Incidence of Surgical Site Infections in Abdominal Closures: a Meta-Analysis. J Gastrointest Surg Off J Soc Surg Aliment Tract. 2017;21(5):896-903. doi:10.1007/s11605-017-3357-6.

6. Webster J, Alghamdi A. Use of plastic adhesive drapes during surgery for preventing surgical site infection. Cochrane Database Syst Rev. 2015;(4):CD006353.

doi:10.1002/14651858.CD006353.pub4.

7. Papaconstantinou HT, Ricciardi R, Margolin DA, et al. A Novel Wound Retractor Combining Continuous Irrigation and Barrier Protection Reduces Incisional Contamination in Colorectal Surgery. World J Surg. 2018;42(9):3000-7. doi:10.1007/s00268-018-4568-z.

8. Bennett-Guerrero E, Pappas TN, Koltun WA, et al. Gentamicin-collagen sponge for infection prophylaxis in colorectal surgery. N Engl J Med. 2010;363(11):1038-49. doi:10.1056/NEJMoa1000837.

9. Ehrlich GD, Hiller NL, Hu FZ. What makes pathogens pathogenic. Genome Biol. 2008;9(6):225. doi:10.1186/gb-2008-9-6-225. 
10. Bhakta A, Tafen M, Glotzer O, et al. Increased Incidence of Surgical Site Infection in IBD Patients. Dis Colon Rectum. 2016;59(4):316-22. doi:10.1097/DCR.0000000000000550.

11. Gurunathan U, Ramsay S, Mitrić G, Way M, Wockner L, Myles P. Association Between Obesity and Wound Infection Following Colorectal Surgery: Systematic Review and Meta-Analysis. J Gastrointest Surg Off J Soc Surg Aliment Tract. 2017;21(10):1700-12. doi:10.1007/s11605-017-3494-y.

12. Nolan MB, Martin DP, Thompson R, Schroeder DR, Hanson AC, Warner DO. Association Between Smoking Status, Preoperative Exhaled Carbon Monoxide Levels, and Postoperative Surgical Site Infection in Patients Undergoing Elective Surgery. JAMA Surg. 2017;152(5):476-83. doi:10.1001/jamasurg.2016.5704.

13. Peponis T, Panda N, Eskesen TG, et al. Preoperative endoscopic retrograde cholangiopancreatography (ERCP) is a risk factor for surgical site infections after laparoscopic cholecystectomy. Am J Surg Published online September 29, 2018. doi:10.1016/j.amjsurg.2018.09.033.

14. Guyton K, Alverdy JC. The gut microbiota and gastrointestinal surgery. Nat Rev Gastroenterol Hepatol. 2017;14(1):43-54. doi:10.1038/nrgastro.2016.139.

15. Byrd AL, Belkaid Y, Segre JA. The human skin microbiome. Nat Rev Microbiol. 2018;16(3):143-55. doi:10.1038/nrmicro.2017.157.

16. Linehan JL, Harrison OJ, Han S-J, et al. Non-classical Immunity Controls Microbiota Impact on Skin Immunity and Tissue Repair. Cell. 2018;172(4):784-96.e18. doi:10.1016/j.cell.2017.12.033.

17. Nakatsuji T, Chen TH, Butcher AM, et al. A commensal strain of Staphylococcus epidermidis protects against skin neoplasia. Sci Adv. 2018;4(2):eaao4502. doi:10.1126/sciadv.aao4502.

18. Nakagawa S, Matsumoto M, Katayama Y, et al. Staphylococcus aureus Virulent PSMa Peptides Induce Keratinocyte Alarmin Release to Orchestrate IL-17-Dependent Skin Inflammation. Cell Host Microbe. 2017;22(5):667-77.e5. doi:10.1016/j.chom.2017.10.008.

19. Wanke I, Steffen H, Christ C, et al. Skin commensals amplify the innate immune response to pathogens by activation of distinct signaling pathways. J Invest Dermatol. 2011;131(2):382-90. doi:10.1038/jid.2010.328.

20. Yang J-J, Chang T-W, Jiang Y, et al. Commensal Staphylococcus aureus Provokes Immunity to Protect against Skin Infection of Methicillin-Resistant Staphylococcus aureus. Int J Mol Sci. 2018;19(5). doi:10.3390/ijms19051290.

21. Paharik AE, Parlet CP, Chung N, et al. Coagulase-Negative Staphylococcal Strain Prevents Staphylococcus aureus Colonization and Skin Infection by Blocking Quorum Sensing. Cell Host Microbe. 2017;22(6):746-56.e5. doi:10.1016/j.chom.2017.11.001.

22. Lai Y, Di Nardo A, Nakatsuji T, et al. Commensal bacteria regulate Toll-like receptor 3-dependent inflammation after skin injury. Nat Med. 2009;15(12):1377-82. doi:10.1038/nm.2062.

23. Bay L, Kragh KN, Eickhardt SR, et al. Bacterial Aggregates Establish at the Edges of Acute Epidermal Wounds. Adv Wound Care. 2018;7(4):105-13. doi:10.1089/wound.2017.0770. 
24. Canesso MCC, Vieira AT, Castro TBR, et al. Skin Wound Healing Is Accelerated and Scarless in the Absence of Commensal Microbiota. J Immunol. 2014;193(10):5171-80. doi:10.4049/jimmunol.1400625.

25. Fleming ID, Krezalek MA, Belogortseva N, et al. Modeling Acinetobacter baumannii wound infections: The critical role of iron. J Trauma Acute Care Surg. 2017;82(3):557-65. doi:10.1097/TA.0000000000001338.

26. Schierle CF, De la Garza M, Mustoe TA, Galiano RD. Staphylococcal biofilms impair wound healing by delaying reepithelialization in a murine cutaneous wound model. Wound Repair Regen Off Publ Wound Heal Soc Eur Tissue Repair Soc. 2009;17(3):354-9. doi:10.1111/j.1524-475X.2009.00489.x.

27. Zhang M, Jiang Z, Li D, et al. Oral antibiotic treatment induces skin microbiota dysbiosis and influences wound healing. Microb Ecol. 2015;69(2):415-21. doi:10.1007/s00248-014-0504-4.

28. Bartow-McKenney C, Hannigan GD, Horwinski J, et al. The microbiota of traumatic, open fracture wounds is associated with mechanism of injury. Wound Repair Regen. 2018;26(2):127-35. doi:10.1111/wrr.12642.

29. Fa-Si-Oen PR, Kroeze F, Verhoef LHM, Verwaest C, Roumen RMH. Bacteriology of abdominal wounds in elective open colon surgery: a prospective study of 100 surgical wounds. Clin Microbiol Infect. 2005;11(2):155-7. doi:10.1111/j.1469-0691.2004.01011.x.

30. Rood KM, Buhimschi IA, Jurcisek JA, et al. Skin Microbiota in Obese Women at Risk for Surgical Site Infection After Cesarean Delivery. Sci Rep. 2018;8(1):8756. doi:10.1038/s41598-018-27134-5.

31. Zeeuwen PL, Boekhorst J, Bogaard EH, van den, et al. Microbiome dynamics of human epidermis following skin barrier disruption. Genome Biol. 2012;13(11):R101. doi:10.1186/gb-2012-13-11-r101.

32. Gomila A, Carratalà J, Camprubí D, et al. Risk factors and outcomes of organ-space surgical site infections after elective colon and rectal surgery. Antimicrob Resist Infect Control. 2017;6:40. doi:10.1186/s13756-017-0198-8.

33. Greco M, Capretti G, Beretta L, Gemma M, Pecorelli N, Braga M. Enhanced recovery program in colorectal surgery: a meta-analysis of randomized controlled trials. World J Surg. 2014;38(6):153141. doi:10.1007/s00268-013-2416-8.

34. Poon JT, Law W-L, Wong IW, et al. Impact of laparoscopic colorectal resection on surgical site infection. Ann Surg. 2009;249(1):77-81. doi:10.1097/SLA.0b013e31819279e3.

35. Ubeda C, Taur Y, Jenq RR, et al. Vancomycin-resistant Enterococcus domination of intestinal microbiota is enabled by antibiotic treatment in mice and precedes bloodstream invasion in humans. J Clin Invest. 2010;120(12):4332-41.

36. Kim S, Covington A, Pamer EG. The intestinal microbiota: Antibiotics, colonization resistance, and enteric pathogens. Immunol Rev. 2017;279(1):90-105. doi:10.1111/imr.12563.

37. Olsan EE, Byndloss MX, Faber F, Rivera-Chávez F, Tsolis RM, Bäumler AJ. Colonization resistance: The deconvolution of a complex trait. J Biol Chem. 2017;292(21):8577-81. doi:10.1074/jbc.R116.752295. 
38. Myles IA, Earland NJ, Anderson ED, et al. First-in-human topical microbiome transplantation with Roseomonas mucosa for atopic dermatitis. JCI Insight. 2018;3(9). doi:10.1172/jci.insight.120608.

39. Partlow J, Blikslager A, Matthews C, et al. Effect of topically applied Saccharomyces boulardii on the healing of acute porcine wounds: a preliminary study. BMC Res Notes. 2016;9. doi:10.1186/s13104016-2012-8.

40. Bode LGM, Kluytmans JAJW, Wertheim HFL, et al. Preventing surgical-site infections in nasal carriers of Staphylococcus aureus. N Engl J Med. 2010;362(1):9-17. doi:10.1056/NEJMoa0808939.

41. Romano-Bertrand S, Frapier J-M, Calvet B, et al. Dynamics of the surgical microbiota along the cardiothoracic surgery pathway. Infect Dis. 2015;5:787. doi:10.3389/fmicb.2014.00787.

42. Castellà L, Sopena N, Rodriguez-Montserrat $D$, et al. Intervention to reduce the incidence of surgical site infection in spine surgery. Am J Infect Contro/ Published online November 7, 2019. doi:10.1016/j.ajic.2019.09.007.

\section{Figures}

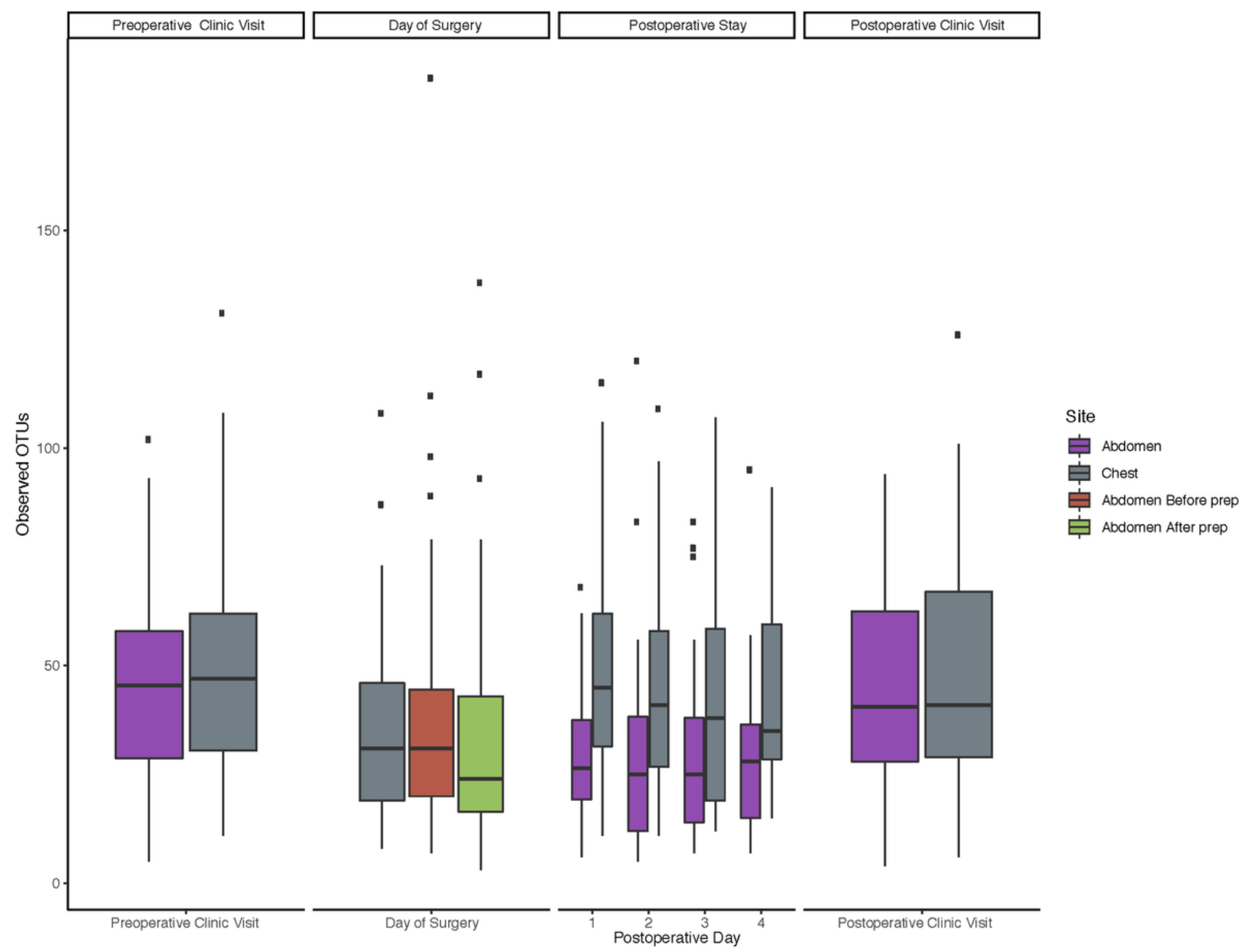


Figure 1

Perioperative variation in alpha diversity present within skin swabs. Shown are the numbers of observed operational taxonomic units (OTUs) in skin swabs from the site of the largest abdominal incision or the chest wall. Values on the x-axis indicate days before(negative) and after surgery. On the day of surgery, the abdominal incision was swabbed before and after skin preparation with chlorhexidine. Those that are significant by Wilcoxon rank-sum test are indicated by an asterisk.
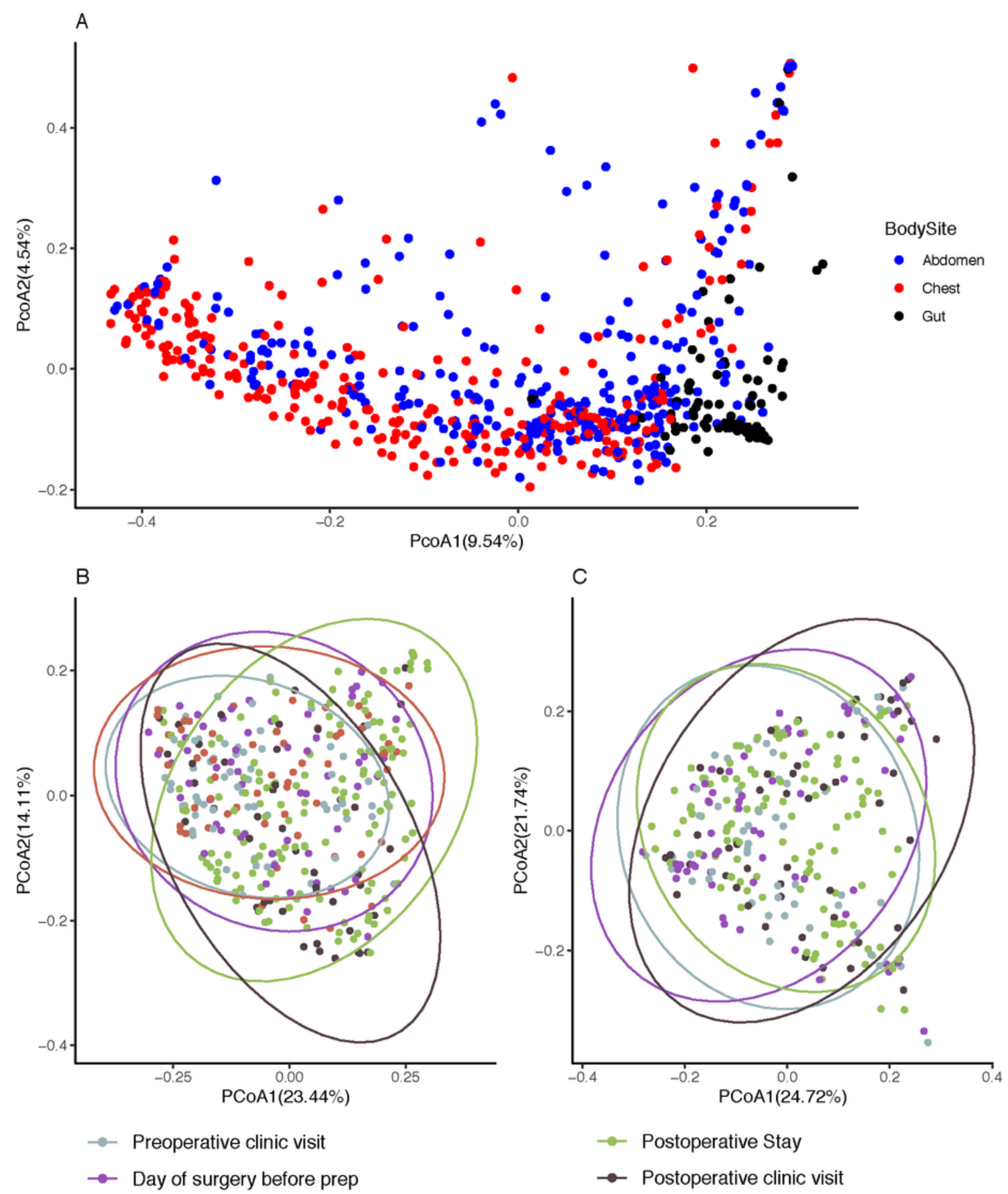

$\rightarrow$ Preoperative clinic visit

$\rightarrow$ Day of surgery before prep

$\rightarrow$ Day of surgery after prep (Abdomen Specific) 


\section{Figure 2}

Perioperative variation in beta diversity present within skin swabs. A) PCoA plot of fecal samples (black), skin swabs from the incision site (blue), and skin swabs from the chest wall (red). Differences between sites were significant by PERMANOVA ( $p$-value<0.001) B) PCoA plot of abdominal skin samples colored by sampling time. C) PCoA plot of chest wall swabs colored by sampling time. Dispersion of data points in $\mathrm{B}$ and $\mathrm{C}$ were evaluated with PERMDISP, quantifying the increased dispersion among abdominal swabs $($ PERMDISP, $p$-value $($ Abdomen $)=0.001, p$-value $($ Chest $)=0.185)$. Permutational multivariate analyses of variance are summarized in Table $\mathrm{S} 1$. 

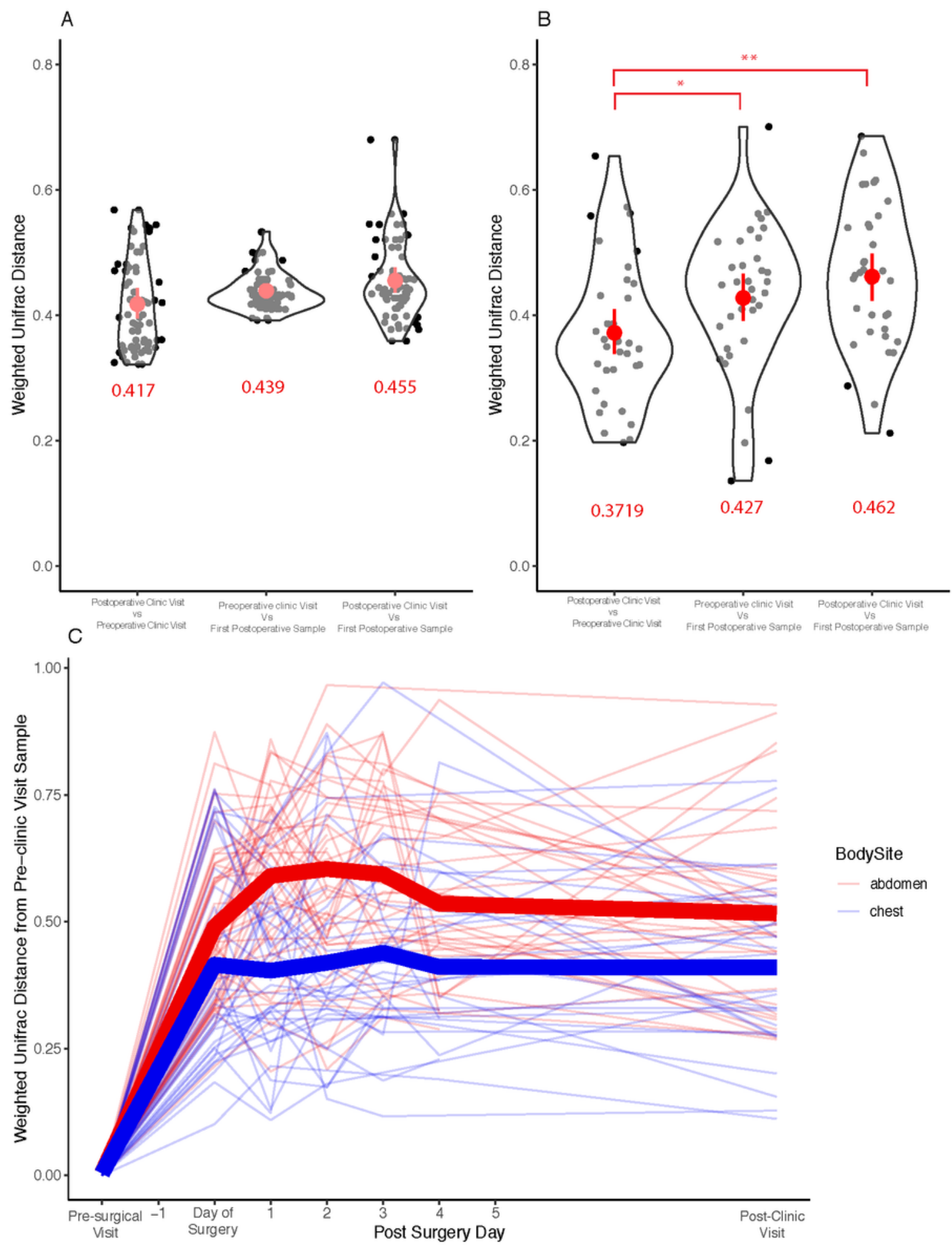

Figure 3

Perioperative variation in pairwise weighted UniFrac distances between incisional skin swabs. A) Average weighted UniFrac distances between all samples from all patients collected during the preoperative clinic visit, the postoperative inpatient stay, and postoperative outpatient clinic visit. B) Intraindividual pairwise distances between samples collected during the preoperative clinic visit, the postoperative inpatient stay, and postoperative outpatient clinic visit. Red brackets indicate significance by Wilcoxon signed-rank test 
$(*<0.05, * *<0.005)$. C) Temporal changes in weighted UniFrac distances between preoperative and postoperative skin swabs of the abdomen (red) and chest wall (black) over time. For each subject, the weighted UniFrac distances between pre-and postoperative samples are plotted against postoperative date.
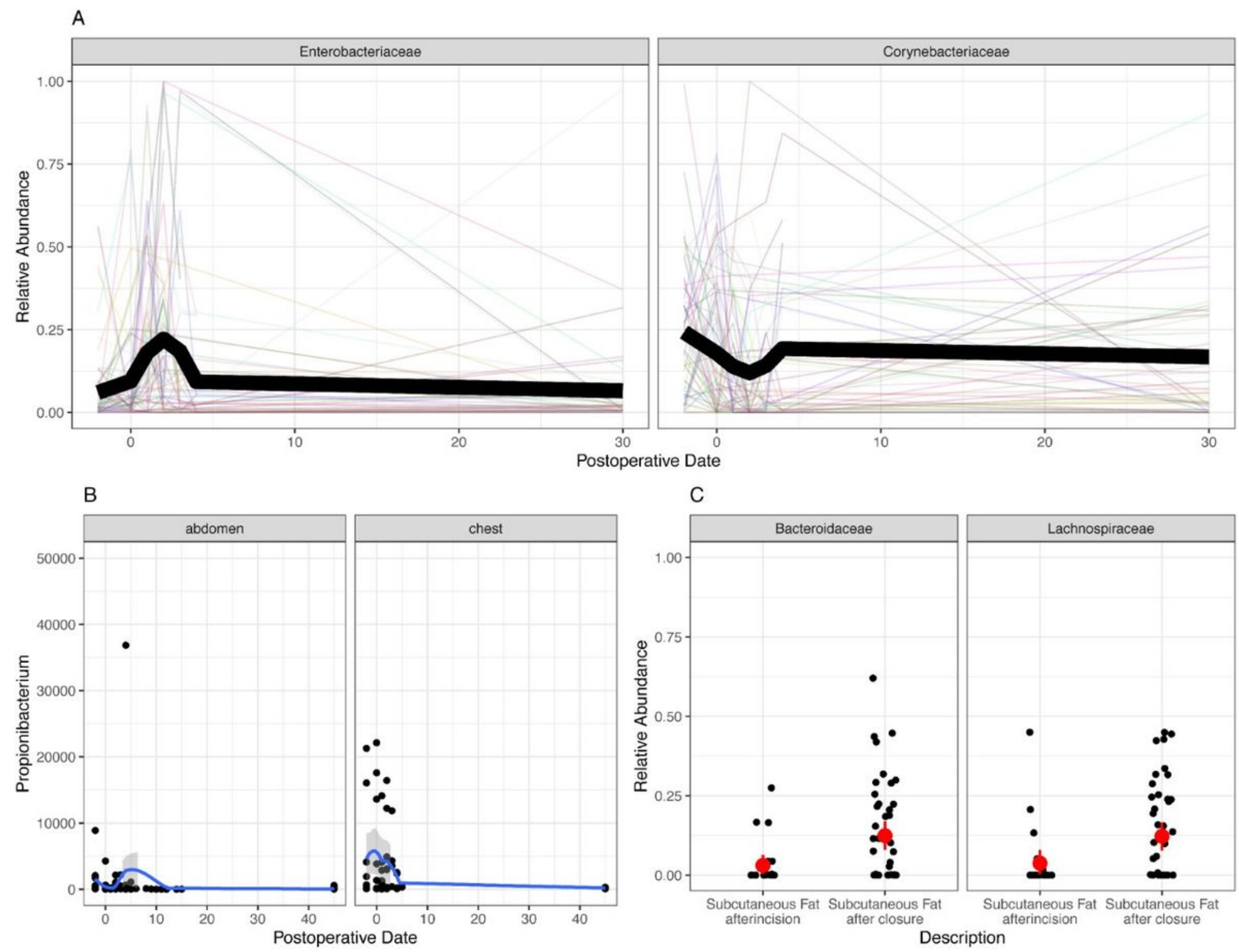

Figure 4

Perioperative variations in taxonomic composition within skin swabs. A) Bacterial families with significant temporal variation in abundance within abdominal swabs. B) Temporal variation in abundance of Propionibacterium on the abdomen and chest, measured by quantitative PCR using Propionibacterium specific primers. C) Compositional differences in sub-cutaneous fat swabs before at time of incision and at time of closure. Predictions of enriched taxa in $\mathrm{A}$ and $\mathrm{C}$ were taken from a consensus of three different prediction methods using a p-value cut-off of 0.05 .

\section{Supplementary Files}


This is a list of supplementary files associated with this preprint. Click to download.

- Onlinetext.docx 